\title{
Professional ethics, VBAC and COVID-19 pandemic: A challenge to be resolved (Review)
}

\author{
ALEXANDRU CARAULEANU, INGRID ANDRADA TANASA, \\ DRAGOS NEMESCU and DEMETRA SOCOLOV
}

Department of Obstetrics and Gynecology, 'Grigore T. Popa' University of Medicine and Pharmacy, 700115 Iasi, Romania

Received May 12, 2021; Accepted June 11, 2021

DOI: $10.3892 /$ etm.2021.10388

\begin{abstract}
Since the declaration of the severe acute respiratory syndrome coronavirus 2 (SARS-CoV-2) pandemic by the World Health Organization (WHO) on March 11, 2020, the entire healthcare system is trying to adapt its capabilities to a challenge that induces a deep and continuous metamorphosis of people, strategies and policies. The right to proper health care is universal, and the patient's autonomy must be respected even in ambiguous times. In the context of increased Cesarean section (CS) rates, the women's desire to achieve vaginal birth after Cesarean section (VBAC) is becoming more articulate, and healthcare professionals need to adapt their approaches regarding the mode of delivery. But how to balance this aspect with respect to the paucity of resources during the pandemic, without infringing the fundamental rights and ethical principles is a demanding question. This article describes a clinical ethical decision-making framework for recommending trial of labor after Cesarean section (TOLAC), and individualized management of VBAC cases tailored upon the new circumstances dictated by the SARS-CoV-2 pandemic.
\end{abstract}

\section{Contents}

1. Introduction

2. Ethical concepts and dilemmas

Correspondence to: Dr Ingrid Andrada Tanasa, Department of Obstetrics and Gynecology, 'Grigore T. Popa' University of Medicine and Pharmacy, 16 Universitatii Street, 700115 Iasi, Romania E-mail: tanasaingrid@yahoo.com

Abbreviations: ACOG, American College of Obstetricians and Gynecologists; AUC, area under the curve; BMI, body mass index; CS, Cesarean section; COVID-19, coronavirus disease 19; SMFM, Society for Maternal-Fetal Medicine; TOLAC, trial of labor after Cesarean; VBAC, vaginal birth after Cesarean; WHO, World Health Organization

Key words: COVID-19, VBAC, TOLAC, professional ethics, beneficence
3. The beneficence-based approach to recommending mode of delivery

4. Fine-tuning the beneficence-based analysis

5. Conclusions

\section{Introduction}

The SARS-CoV-2 pandemic, and the global response to it, has induced extraordinary circumstances that has led to profound adaptive changes in various domains, especially in the healthcare system. The field of obstetrics is no exception, and numerous recommendations have determined fundamental shifts regarding system protocols, attitudes and responses. But any change of strategy needs time for the documentation of long-term effects, especially for outlining the adverse ones related to specific cases.

Professional ethics provides essential guidance to obstetricians for offering, recommending, and performing Cesarean section (CS) or vaginal birth after Cesarean section (VBAC), at the same time trying to emphasize the ethical dilemmas and moral implications of different obstetrical actions, especially in the grey area created by this pandemic.

The VBAC rate has shown an increasing trend, mainly in developed countries, so that the statistics for 2018 indicate a rate of $13.3 \%$ in the USA (1). Worldwide, there is a heterogenous distribution of VBAC rates, ranging from 20 to $55 \%$ in Europe $(2,3)$. However, a reduction in these rates is expected due to the coronavirus disease-19 (COVID-19) pandemic.

In contrast, the rates for CS continue to rise, exceeding the WHO recommendation of an ideal CS rate between $10-15 \%$, so that some countries have reached a $50 \%$ or more CS rate, especially in poor or developing countries $(4,5)$. This phenomenon raises concerns about the health implications, work ethics, inequality, discrimination, and healthcare costs.

In order to reduce the important variation in the clinical management of pregnant women who desire to achieve VBAC during the period of the SARS-CoV-2 pandemic, this article describes a clinical ethical decision-making framework for recommending trial of labor after Cesarean section (TOLAC), and individualized management of VBAC cases.

The proposed clinical ethical framework emphasizes the importance of a beneficence principle by identifying clinical factors that may increase or decrease the risk of significant 
maternal and fetal harm. Moreover, this article illustrates the implementation of this framework into the decision-making process concerning TOLAC, aiming to improve the quality of obstetrical care.

\section{Ethical concepts and dilemmas}

When deciding the mode of delivery, it is important for the obstetrician to consider clinically relevant ethical principles and their application to patient care. The ethical principles of beneficence and non-maleficence, which require the clinician to seek the best balance of clinical good over clinical harm in the management of the patient, should be fundamentals for every medical decision.

In the case of Cesarean delivery as a therapeutic measure, maternal nonmaleficence poses limits to maternal and fetal beneficence. The pregnant woman who undergoes Cesarean delivery is submitting herself to an invasive abdominal surgery that can impose significant harm.

The short-term risks of CS include postoperative pain, injury to adjacent organs, wound infection, longer recovery time, and rarely death. The long-term risks associated with CS include skin and uterine scarring (which in turn would increase the risk of abnormal placentation), elective repeat Cesarean delivery (ERCD) for future pregnancies, and postpartum depression (6).

As for fetal outcome, unindicated Cesarean delivery before term is associated with an increased risk of transient tachypnea, respiratory distress syndrome, and persistent pulmonary hypertension (7).

Another important ethical principle for the obstetricians to consider is respect for a patient's autonomy, which requires the physician to empower the pregnant woman with the clinical information that she needs in order to make informed decisions regarding the benefits and risks of different modes of delivery.

In obstetrical practice, respecting the pregnant patient's autonomy implies the recognition of her decisional rights about the management of her pregnancy and birth. Therefore, the woman's informed consent for Cesarean delivery or VBAC should be obtained after proper counseling.

Under the new guidelines, the American College of Obstetricians and Gynecologists (ACOG) reiterates its commitment to patient autonomy, and reaffirms a woman's right to choose VBAC (8). Yet, in practice, liability issues have a negative impact over the physicians and facilities compliance with ACOG recommendations.

Moreover, the SARS-CoV-2 pandemic has brought to our attention the moral distress concept, which was initially mentioned in nursing ethics, and expanded to all health professions. This concept is illustrated by situations in which a healthcare professional believes he or she knows the morally right thing to do, but is unable to do so $(9,10)$. Among the sources of moral distress, institutional policies, and decisional hierarchies were cited (11), and it becomes obvious that health policies and controversial statements put additional stress on this matter.

As for ethical dilemmas, their main substrate is the battle between the professional responsibility, and circumstances induced by the new pandemic.

Do obstetricians have the professional responsibility to treat a COVID-19-positive patient regardless of the personal risk of infection? It is true that protective equipment, when used correctly, prevents exposure to the virus, and limits the infection. On the other hand, numerous healthcare facilities encountered a scarcity of protective resources, especially at the beginning of the pandemic (12), and high-risk categories of healthcare providers (those over 60 years of age, with comorbidities, pregnant women) were vulnerable while caring for infected patients. A solution for this is ensuring that every hospital has proper protective gear, and that the personnel is instructed regarding the specific COVID-19 protocols.

How to approach an VBAC case positive for COVID-19 when there is a shortage of trained personnel and resources is an urgent issue. Candidates that are appropriate for TOLAC, but have been confirmed positive for the new coronavirus, are facing limitations when choosing a mode of delivery. The paucity of information regarding the vertical transmission of the virus, the need for continuous monitoring during labor, and in case of adverse obstetrical events such as uterine rupture, emergency CS, are factors that need to be considered when recommending TOLAC. However, the principle of autonomy must be respected, and if appropriate, a proper counselling, followed by an informed decision can solve this dilemma.

\section{The beneficence-based approach to recommending mode of delivery}

Obstetricians should employ a beneficence-based approach when recommending a specific mode of delivery, and should consider the factors associated with significant maternal and fetal harm.

The first factor to be considered is the availability of TOLAC at the planned healthcare unit. ACOG recommends conducting TOLAC at facilities that are able to perform an emergency CS when it is required (13), but in some cases women may choose to pursue TOLAC even at hospitals with limited resources. This sensitive option may further lead to liability issues, but it must not be considered a reason for limitation of access to healthcare. A solution to this situation may be discussing birthing options early in pregnancy, and transfer of care to a better prepared healthcare facility, while developing internal hospital protocols that are aligned with the international and local guidelines and regulations. Anyway, we emphasize that TOLAC should never be attempted at home.

The guidelines emitted by ACOG, the Society for Maternal-Fetal Medicine (SMFM), and others include general recommendations (available at acog.org and SMFM. org) regarding prevention, diagnosis, and case management of COVID-19-positive patients, as well as suggestions for modifying traditional protocols for prenatal visits. These modifications include telehealth, reducing the number of in-person visits, timing of visits, restricting visitors during visits and tests, timing of indicated obstetric ultrasound examinations or other prenatal tests. However, after lockdown, the stillbirth rate has apparently increased, an aspect which may have been caused by disruptions in prenatal care, and by a higher number of home births (14-17).

The SARS-CoV-2 pandemic has led to a separation of hospitals into two categories: Those who can receive confirmed cases, and those who act as a tampon until proper transfer of 
the patients. This segregation may further limit the women's access to immediate life-saving procedures due to personnel and equipment shortage (18).

The second factor to be considered is the probability of achieving a VBAC. The success rates are highly variable among countries, healthcare facilities and obstetricians, while patient characteristics and non-medical factors may influence the likelihood of successful TOLAC (19). For example, in the USA, the success rate of TOLAC was estimated in 2013 at $70.4 \%$ for women who had one previous Cesarean delivery, and at $51.4 \%$ for those with two or more prior Cesarean deliveries; thus, the statistics are encouraging (20).

Moreover, a previous vaginal delivery was found to be associated with increased likelihood of successful TOLAC (21), while the most important factor that precluded VBAC was a prior Cesarean delivery for a recurring indication, such as failure to progress. Induction of labor significantly reduced the odds of VBAC (13).

Many patients and physicians now have access to different calculators that offer an individualized estimation regarding the likelihood of a successful TOLAC. These results, combined with a proper counseling session that includes the risks and benefits associated with a TOLAC, specific circumstances of the prior CS, anticipated weight of the fetus, desire for future childbearing, and other individual level factors that may influence the chance of success, are powerful beneficence-based approaches that can be included into the current practice. However, the main problem with the calculators is the validation into different situations (e.g., multiple gestations).

The most utilized calculator is the Maternal-Fetal Medicine Units Network (MFMU) that predicts the chance of VBAC based on data available at the first prenatal visit: Maternal age, height, weight, pre-pregnancy body mass index (BMI), race, ethnicity, prior vaginal delivery, prior VBAC, and prior Cesarean for arrest of dilation or descent (22). This calculator has been validated in women with one or two prior Cesarean deliveries (23), and its overall predictive capability was found to be good, with an area under the curve (AUC) of 0.75 (95\% CI 0.74-0.77) (24).

The MFMU Network website also provides a VBAC calculator that predicts the chance of VBAC based on information available at the time of admission for delivery (25): Height and weight, BMI at delivery, gestational age, cervical examination, preeclampsia/gestational hypertension, and labor induction. The overall AUC for this model was 0.77 (95\% CI 0.76-0.78) (26). It was externally validated in independent cohorts of women with one prior Cesarean delivery (27).

For preterm delivery, between $26+0$ and $36+6$ weeks of gestation, a recent model estimated the VBAC likelihood at $76.6 \%$, which was positively influenced by diabetes, greater cervical dilation, history of vaginal birth, and history of VBAC, while induction of labor, recurring indication for prior Cesarean, and hypertensive disease had a negative influence over the VBAC rate. The overall predictive capability of the model was good with an AUC of $0.80(95 \%$ CI 0.77-0.83) (28).

Considering these instruments, VBAC should be recommended when there are weak or no beneficence-based obligations to the pregnant woman and to the fetus, while ERCD would be recommended when these aspects are pertinent, and the grey area defined by intermediate beneficence-based obligations should impose a careful and multidisciplinary approach of the case before strongly recommending a mode of delivery.

The third factor that must be considered is the woman's preference. A physician must respect the patient's desire regarding the route of delivery, and must take into consideration the values and opinions expressed by the pregnant women.

The conflictual existing data with respect to vertical transmission of COVID-19 (29-31), viral complications $(32,33)$, and pregnancy outcomes for infected patients $(34,35)$ may reduce the women's desire to achieve VBAC, but the obstetrician's role must be a reassuring one, and must guide the patients through the avalanche of new information, so that the patient can consciously choose a mode of delivery.

\section{Fine-tuning the beneficence-based analysis}

As part of the ethical decision-making framework for recommending the mode of delivery, the obstetrician should fine-tune his or her beneficence-based analysis by identifying the most suitable TOLAC candidates.

An ideal candidate for TOLAC will have a high likelihood of VBAC, and a very low likelihood of intrapartum major complications such as uterine rupture (Table I).

TOLAC seems to be a reasonable option for women who have undergone only one previous CS via a low transverse hysterotomy (36), with an estimated VBAC rate between 60 and $80 \%$ (37), and a uterine rupture rate of $0.4-0.7 \%$ (19).

The obstetrician must determine the extent into the contractile portion of the uterus of a prior low vertical uterine incision, when considering TOLAC. If the previous hysterotomy did not reach the contractile portion of the uterus, then the patient must be informed about the risks associated with TOLAC, including uterine rupture, which in this case is approximated at $1-2 \%$ (37).

Multiple Cesarean sections performed via low transverse hysterotomy may lead to an increased risk of uterine rupture (38), but these women may be candidates for TOLAC after individualized counseling that takes into consideration other factors that predict the likelihood of successful VBAC (13). The VBAC rates appear to be similar among patients who have had two or three prior Cesarean deliveries (VBAC-2 vs. VBAC-3: 71.1 vs. $75.5 \%)(38,39)$.

In the case of an unknown type of uterine incision, ACOG supports the assumption that most women with a prior CS have a low transverse hysterotomy, and therefore TOLAC should be a reasonable option for them (13). Two studies concluded that women who have undergone an unknown type of prior incision had a similar rate of uterine rupture as those with a known prior low transverse uterine incision $(40,41)$.

ACOG does not consider a contraindication for TOLAC at a gestational age beyond 40 weeks of gestation (13), although several studies outlined a lower VBAC rate for this type of candidates $(42,43)$. A secondary analysis compared the obstetric outcomes between TOLAC candidates undergoing induction of labor and those undergoing expectant 
Table I. Pregnant patient characteristics that are indicative or not for TOLAC.

Suitable TOLAC indications

Poor TOLAC indication

One prior low transverse uterine incision

Prior low vertical uterine incision

Two prior low transverse uterine incisions

Three prior low transverse uterine incisions

Unknown type of uterine incision

Pregnancy more than 40 weeks of gestation

Twin gestation

Macrosomia

Obesity

TOLAC, trial of labor after Cesarean.

management $\geq 39$ weeks of gestation, and found that labor induction at 39 weeks was associated with a higher VBAC rate than expectant management (74 vs. 61\%), and that the risk of uterine rupture was also higher (1.4 vs. $0.5 \%$ ) (44).

According to ACOG, twin gestation and suspected fetal macrosomia (estimated fetal weight $\geq 4,000 \mathrm{~g}$ ) are not contraindications for TOLAC, as long as there are no other factors that preclude vaginal delivery (13). The past birth weight(s), outcomes, and the predicted birth weight in the current pregnancy are key elements when recommending TOLAC.

When the physician estimates a fetal weight of more that 4,000 $\mathrm{g}$, and the woman has had no previous vaginal delivery, the expected VBAC rate is lower, being estimated at less than $50 \%$ in some studies $(45,46)$. Also, the adverse obstetrical outcomes, such as uterine rupture are more frequently encountered in these cases $(47,48)$. Thus, a previous vaginal delivery is an important factor to be considered when recommending TOLAC for women with macrosomic fetuses.

Maternal obesity (BMI $\geq 30 \mathrm{~kg} / \mathrm{m}^{2}$ ) should not be considered a contraindication for TOLAC when other factors positively indicate a trial of labor and vaginal delivery. However, some studies have concluded that a successful TOLAC is less likely to be achieved by obese patients $(24,49-51)$.

In a secondary analysis from a prospective observational study, the authors identified a rate of failed trial of labor of $15 \%$ in normal-weight women, $30 \%$ in obese women (BMI $30-39.9 \mathrm{~kg} / \mathrm{m}^{2}$ ), and $39 \%$ in severely obese women $\left(\mathrm{BMI} \geq 40 \mathrm{~kg} / \mathrm{m}^{2}\right)$, with a uterine dehiscence/rupture rate of $0.9,1.4$, and $2.1 \%$ for the three mentioned groups (52). Thus, a careful monitoring of TOLAC in obese patients is recommended.

ACOG recommends ERCD for women with prior trans-fundal uterine incision, prior uterine rupture, and for women with standard contraindications to labor or vaginal birth (eg, placenta previa) (13).

In a literature review by Sabol et al, the frequency of uterine rupture for classical or T-shaped incisions ranged from 4 to $9 \%$ (37), which exceeds the estimated rates for low vertical incisions and low transverse uterine incisions Moreover, in another study the uterine rupture rate for women with prior classical, inverted $\mathrm{T}$ or $\mathrm{J}$ incision was $1.9 \%$ (40).
As for trans-myometrial incisions, the uterine dehiscence/rupture rate was estimated at $14 \%$, which is unacceptably high (53).

Women with a previous uterine rupture confined to the lower uterine segment are reported to have a $6 \%$ recurrence rate of the uterine rupture during labor, while for those in whom the prior uterine rupture involved the contractile segment, the recurrence rate was estimated at $32 \%$ (54).

If an adverse obstetric outcome such as uterine rupture or non-reassuring fetal rate occurs during TOLAC, then the obstetrician must immediately change the case management strategy after a proper evaluation of the risks.

With the emergence of new vaccines and vaccination campaigns, a reduction in the pandemic duration can be expected, although the long-term protection offered by vaccines against SARS-CoV-2 and its new strains is still under evaluation (55). Thus, all healthcare facilities must adapt to this pleiomorphic epidemiologic context.

The proposed ethical framework requires the organizational capacity to respond to obstetric emergencies, so that TOLAC must be offered only in fully equipped and prepared healthcare facilities.

\section{Conclusions}

The proposed clinical ethical framework outlines a multifactorial approach to fine-tuning the decision-making process for women who desire to achieve VBAC during the COVID-19 pandemic.

This multifactorial approach stresses the importance of the organizational resources and ethical conduits that in addition to the obstetrician's training, experience, and skill level, have a positive effect on the VBAC experience.

Finally, adopting this clinical ethical framework in practice will enhance the autonomy of pregnant women by providing comprehensive counseling about the COVID-19 effects in pregnancy, clinical decisions, and individualized case management.

\section{Acknowledgements}

Not applicable. 


\section{Funding}

No funding was received.

\section{Availability of data and materials}

All information is documented by relevant resources.

\section{Authors' contributions}

$\mathrm{AC}$ and IAT designed the review; AC drafted the initial manuscript. DN and DS carried out the literature search, supported by AC and IAT, who retrieved the evidence and selected the papers. AC, IAT and DS extracted the data; IAT, $\mathrm{AC}, \mathrm{DN}$, and DS participated in writing the final version of the manuscript. IAT and AC were assigned to perform a final check of the manuscript. All authors read and approved the final manuscript.

\section{Ethics approval and consent to participate}

Not applicable.

\section{Patient consent for publication}

Not applicable.

\section{Competing interests}

The authors declare that they have no competing interests.

\section{References}

1. Osterman MJK: Recent Trends in vaginal birth after cesarean delivery: United states, 2016-2018. NCHS Data Brief: 1-8, 2020.

2. Lundgren I, Smith V, Nilsson C, Vehvilainen-Julkunen K Nicoletti J, Devane D, Bernloehr A, van Limbeek E, Lalor J and Begley $\mathrm{C}$ : Clinician-centred interventions to increase vaginal birth after caesarean section (VBAC): A systematic review. BMC Pregnancy Childbirth 15: 16, 2015.

3. Euro PERISTAT: European Perinatal Health Report: The health and care of pregnant women and their babies in 2010. EURONEOSTAT, 2013. https://www.europeristat.

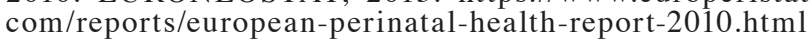
Acessed September 9, 2020.

4. Organisation for Economic Cooperation and Development (OECD): Health at a Glance 2019: OECD Indicators, OECD Publishing, Paris, 2019.

5. Cavallaro FL, Cresswell JA, França GV, Victora CG, Barros AJ and Ronsmans C: Trends in caesarean delivery by country and wealth quintile: Cross-sectional surveys in southern Asia and sub-Saharan Africa. Bull World Health Organ 91: 914D-922D, 2013.

6. Quinlan JD and Murphy NJ: Cesarean delivery: Counseling issues and complication management. Am Fam Physician 91: 178-184, 2015 .

7. No authors listed: ACOG committee opinion no. 559: Cesarean delivery on maternal request. Obstet Gynecol 121: 904-907, 2013.

8. No authors listed: ACOG Practice bulletin no. 115: Vaginal birth after previous cesarean delivery. Obstet Gynecol 116: 450-463, 2010.

9. Jameton A: Nursing practice: The ethical issues. 1984. Englewood Cliffs, NJ, Prentice-Hall, pp331, 1984.

10. Hamric AB, Borchers CT and Epstein EG: Development and testing of an instrument to measure moral distress in healthcare professionals. AJOB Prim Res 3: 1-9, 2012.

11. Hunt M, Chénier A, Bezanson K, Nouvet E, Bernard C, de Laat S, Krishnaraj G and Schwartz L: Moral experiences of humanitarian health professionals caring for patients who are dying or likely to die in a humanitarian crisis. J Int Humanit Action 3: 1-13, 2018.
12. Melman GJ, Parlikad AK and Cameron EAB: Balancing scarce hospital resources during the COVID-19 pandemic using discrete-event simulation. Health Care Manag Sci 9: 1-19, 2021.

13. No authors listed: ACOG Practice Bulletin No. 205: Vaginal birth after cesarean delivery. Obstet Gynecol 133: e110-e127, 2019.

14. Khalil A, von Dadelszen P, Draycott T, Ugwumadu A, O'Brien P and Magee L: Change in the incidence of stillbirth and preterm delivery during the COVID-19 pandemic. JAMA 324: 705-706, 2020.

15. Reale SC, Fields KG, Lumbreras-Marquez MI, King CH, Burns SL, Huybrechts KF and Bateman BT: Association between number of in-person health care visits and SARS-CoV-2 infection in obstetrical patients. JAMA 324: 1210-1212, 2020.

16. Ashish KC, Gurung R, Kinney MV, Sunny AK, Moinuddin M, Basnet O, Paudel P, Bhattarai P, Subedi K, Shrestha MP, et al: Effect of the COVID-19 pandemic response on intrapartum care, stillbirth, and neonatal mortality outcomes in Nepal: A prospective observational study. Lancet Glob Health 8: e1273-e1281, 2020.

17. Kumari V, Mehta K and Choudhary R: COVID-19 outbreak and decreased hospitalisation of pregnant women in labour. Lancet Glob Health 8: e1116-e1117, 2020.

18. Docea AO, Tsatsakis A, Albulescu D, Cristea O, Zlatian O, Vinceti M, Moschos SA, Tsoukalas D, Goumenou M, Drakoulis N, et al: A new threat from an old enemy: Re-emergence of coronavirus (Review). Int J Mol Med 45: 1631-1643, 2020.

19. Guise JM, Eden K, Emeis C, Denman MA, Marshall N, Fu RR, Janik R, Nygren P, Walker M and McDonagh M: Vaginal birth after cesarean: New insights. Evid Rep Technol Assess (Full Rep): 1-397, 2010.

20. Curtin SC, Gregory KD, Korst LM and Uddin SF: Maternal morbidity for vaginal and cesarean deliveries, according to previous cesarean history: New data from the birth certificate, 2013. Natl Vital Stat Rep 64: 1-13, 2015.

21. Eden KB, McDonagh M, Denman MA, Marshall N, Emeis C Fu R, Janik R, Walker M and Guise JM: New insights on vaginal birth after cesarean: Can it be predicted? Obstet Gynecol 116: 967-981, 2010.

22. Maternal-Fetal Medicine Units Network (MFMU): Vaginal birth after cesarean[calculator]. https://mfmunetwork.bscgwuedu/PublicBSC/MFMU/VGBirthCalc/vagbirthhtml. Accessed October 10, 2020.

23. Metz TD, Allshouse AA, Faucett AM and Grobman WA: Validation of a vaginal birth after cesarean delivery prediction model in women with two prior cesarean deliveries. Obstet Gynecol 125: 948-952, 2015.

24. Grobman WA, Lai Y, Landon MB, Spong CY, Leveno KJ, Rouse DJ, Varner MW, Moawad AH, Caritis SN, Harper M, et al: Development of a nomogram for prediction of vaginal birth after cesarean delivery. Obstet Gynecol 109: 806-812, 2007.

25. Maternal-Fetal Medicine Units Network (MFMU): Vaginal birth after cesarean[admission.calculator].https://mfmunetworkbscgwuedu/PublicBSC/MFMU/VGBirthCalc/vagbrth2html. Accessed September 9, 2020.

26. Grobman WA, Lai Y, Landon MB, Spong CY, Leveno KJ, Rouse DJ, Varner MW, Moawad AH, Simhan HN, Harper M, et al: Does information available at admission for delivery improve prediction of vaginal birth after cesarean? Am J Perinatol 26: 693-701, 2009.

27. Costantine MM, Fox KA, Pacheco LD, Mateus J, Hankins GD, Grobman WA and Saade GR: Does information available at delivery improve the accuracy of predicting vaginal birth after cesarean? Validation of the published models in an independent patient cohort. Am J Perinatol 28: 293-298, 2011.

28. Mardy AH, Ananth CV, Grobman WA and Gyamfi-Bannerman C: A prediction model of vaginal birth after cesarean in the preterm period. Am J Obstet Gynecol 215: 513.e1-e7, 2016.

29. Dong L, Tian J, He S, Zhu C, Wang J, Liu C and Yang J: Possible vertical transmission of SARS-CoV-2 from an infected mother to her newborn. JAMA 323: 1846-1848, 2020.

30. Vivanti AJ, Vauloup-Fellous C, Prevot S, Zupan V, Suffee C, Do Cao J, Benachi A and De Luca D: Transplacental transmission of SARS-CoV-2 infection. Nat Commun 11: 3572, 2020.

31. ParazziniF, Bortolus R, Mauri PA, Favilli A, Gerli S and Ferrazzi E: Delivery in pregnant women infected with SARS-CoV-2: A fast review. Int J Gynaecol Obstet 150: 41-46, 2020.

32. Narang K, Enninga EAL, Gunaratne MDSK, Ibirogba ER, Trad ATA, Elrefaei A, Theiler RN, Ruano R, Szymanski LM, Chakraborty R and Garovic VD: SARS-CoV-2 infection and COVID-19 during pregnancy: A multidisciplinary review. Mayo Clin Proc 95: 1750-1765, 2020. 
33. Teles Abrao Trad A, Ibirogba ER, Elrefaei A, Narang K, Tonni G, Picone O, Suy A, Carreras Moratonas E, Kilby MD and Ruano R: Complications and outcomes of SARS-CoV-2 in pregnancy: Where and what is the evidence? Hypertens Pregnancy 39: 361-369, 2020.

34. Di Mascio D, Khalil A, Saccone G, Rizzo G, Buca D, Liberati M, Vecchiet J, Nappi L, Scambia G, Berghella V and D'Antonio F: Outcome of coronavirus spectrum infections (SARS, MERS, COVID-19) during pregnancy: A systematic review and meta-analysis. Am J Obstet Gynecol MFM 2: 100107, 2020.

35. Knight M, Bunch K, Vousden N, Morris E, Simpson N, Gale C, O'Brien P, Quigley M, Brocklehurst P and Kurinczuk JJ; UK obstetric surveillance system SARS-CoV-2 infection in pregnancy collaborative group: Characteristics and outcomes of pregnant women admitted to hospital with confirmed SARS-CoV-2 infection in UK: National population based cohort study. BMJ 369: m2107, 2020.

36. No authors listed: National Institutes of Health Consensus Development conference statement: Vaginal birth after cesarean: New insights March 8-10, 2010. Obstet Gynecol 115: 1279-1295, 2010.

37. Sabol B, Denman MA and Guise JM: Vaginal birth after cesarean: An effective method to reduce cesarean. Clin Obstet Gynecol 58: 309-319, 2015

38. Tahseen $\mathrm{S}$ and Griffiths $\mathrm{M}$ : Vaginal birth after two caesarean sections (VBAC-2)-a systematic review with meta-analysis of success rate and adverse outcomes of VBAC-2 versus VBAC-1 and repeat (third) caesarean sections. BJOG 117: 5-19, 2010.

39. Cahill AG, Tuuli M, Odibo AO, Stamilio DM and Macones GA Vaginal birth after caesarean for women with three or more prior caesareans: Assessing safety and success. BJOG 117: 422-427, 2010.

40. Landon MB, Hauth JC, Leveno KJ, Spong CY, Leindecker S, Varner MW, Moawad AH, Caritis SN, Harper M, Wapner RJ, et al: Maternal and perinatal outcomes associated with a trial of labor after prior cesarean delivery. N Engl J Med 351: 2581-2589, 2004

41. Smith D, Stringer E, Vladutiu CJ, Zink AH and Strauss R: Risk of uterine rupture among women attempting vaginal birth after cesarean with an unknown uterine scar. Am J Obstet Gynecol 213: 80.e1-80.e5, 2015.

42. Zelop CM, Shipp TD, Cohen A, Repke JT and Lieberman E: Trial of labor after 40 weeks' gestation in women with prior cesarean. Obstet Gynecol 97: 391-393, 2001.

43. Coassolo KM, Stamilio DM, Paré E, Peipert JF, Stevens E, Nelson DB and Macones GA: Safety and efficacy of vaginal birth after cesarean attempts at or beyond 40 weeks of gestation. Obstet Gynecol 106: 700-706, 2005.

44. Palatnik A and Grobman WA: Induction of labor versus expectant management for women with a prior cesarean delivery. Am J Obstet Gynecol 212: 358.e1-e6, 2015.
45. Elkousy MA, Sammel M, Stevens E, Peipert JF and Macones G The effect of birth weight on vaginal birth after cesarean delivery success rates. Am J Obstet Gynecol 188: 824-830, 2003.

46. Flamm BL and Goings JR: Vaginal birth after cesarean section: Is suspected fetal macrosomia a contraindication? Obstet Gynecol 74: 694-697, 1989.

47. Zelop CM, Shipp TD, Repke JT, Cohen A and Lieberman E: Outcomes of trial of labor following previous cesarean delivery among women with fetuses weighing $>4000 \mathrm{~g}$. Am J Obstet Gynecol 185: 903-905, 2001.

48. Jastrow N, Roberge S, Gauthier RJ, Laroche L, Duperron L, Brassard N and Bujold E: Effect of birth weight on adverse obstetric outcomes in vaginal birth after cesarean delivery. Obstet Gynecol 115: 338-343, 2010.

49. Landon MB, Leindecker S, Spong CY, Hauth JC, Bloom S, Varner MW, Moawad AH, Caritis SN, Harper M, Wapner RJ, et al: The MFMU cesarean registry: Factors affecting the success of trial of labor after previous cesarean delivery. Am J Obstet Gynecol 193: 1016-1023, 2005.

50. Regan J, Keup C, Wolfe K, Snyder C and DeFranco E: Vaginal birth after cesarean success in high-risk women: A population-based study. J Perinatol 35: 252-257, 2015.

51. Chauhan SP, Magann EF, Carroll CS, Barrilleaux PS, Scardo JA and Martin JN Jr: Mode of delivery for the morbidly obese with prior cesarean delivery: Vaginal versus repeat cesarean section. Am J Obstet Gynecol 185: 349-354, 2001.

52. Hibbard JU, Gilbert S, Landon MB, Hauth JC, Leveno KJ, Spong CY, Varner MW, Caritis SN, Harper M, Wapner RJ, et al: Trial of labor or repeat cesarean delivery in women with morbid obesity and previous cesarean delivery. Obstet Gynecol 108: 125-133, 2006.

53. Wilson RD, Lemerand K, Johnson MP, Flake AW, Bebbington M, Hedrick HL and Adzick NS: Reproductive outcomes in subsequent pregnancies after a pregnancy complicated by open maternal-fetal surgery (1996-2007). Am J Obstet Gynecol 203: 209.e1-e6, 2010.

54. Ritchie EH: Pregnancy after rupture of the pregnant uterus. A report of 36 pregnancies and a study of cases reported since 1932. J Obstet Gynaecol Br Commonw 78: 642-648, 1971.

55. Calina D, Docea AO, Petrakis D, Egorov AM, Ishmukhametov AA, Gabibov AG, Shtilman MI, Kostoff R, Carvalho F, Vinceti M, et al: Towards effective COVID-19 vaccines: Updates, perspectives and challenges (Review). Int J Mol Med 46: 3-16, 2020.

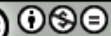

This work is licensed under a Creative Commons Attribution-NonCommercial-NoDerivatives 4.0 International (CC BY-NC-ND 4.0) License. 